\title{
Estudio estadístico de la educación vial en las Unidades Educativas de la Ciudad de Riobamba
}

\section{Statistical study of road education in the educational units of the city of Riobamba}

Fabián Patricio Londo Yachambáy. ${ }^{1}$, Narcisa de Jesús Sánchez Salcán. ${ }^{2}$, Adriana Margarita Morales Noriega. ${ }^{3} \&$ Xavier Alejandro Guerra Sarche. ${ }^{4}$

\begin{abstract}
. DOI: https://doi.org/10.33262/cienciadigital.v3i3.624

This research study was made to high school students of the Educational Units of the canton of Riobamba, who are a vulnerable point in the accident issue, the objective of the research is to analyze the knowledge that this population under study has about what is Road Safety Education. A quantitative approach was applied, the types of research used were bibliographic and descriptive, supported by the inductive-deductive and analytic-synthetic and deductive methods; the techniques used to collect primary information correspond to the survey applied to high school students. The results of the investigation showed that this population is an entity that has low knowledge of what is Road Safety, so there is no adequate training in schools on this vital issue, as well as a disinterest in improving this situation, weaknesses with a lack of adequate signage. To overcome these shortcomings, it is imperative to implement a subject on road education at different levels of education to design communication strategies, in order to raise awareness among all citizens on the theme of First Pedestrian, in order to

${ }^{1}$ Escuela Superior Politécnica de Chimborazo, Facultad de Administración de Empresas. Riobamba, Ecuador. flondo@espoch.edu.ec

${ }^{2}$ Universidad Nacional de Chimborazo. Facultad de Ciencias de la Educación Humanas y Tecnologías, nsanchez@unach.edu.ec

${ }^{3}$ Escuela Superior Politécnica de Chimborazo, Facultad de Administración de Empresas. Riobamba, Ecuador. adriana.morales@espoch.edu.ec

${ }^{4}$ Escuela Superior Politécnica de Chimborazo, Facultad de Administración de Empresas. Riobamba, Ecuador. xavier.guerra@espoch.edu.ec
\end{abstract}


generate identity and project a favorable image and thus reduce the high accident rates that are in the country.

Keywords: Road safety education, Statistical analysis, Traffic accidents, Signage

\section{Resumen.}

El presente estudio de investigación se realizó a estudiantes de Bachillerato de las Unidades Educativas del cantón Riobamba, quienes son un punto vulnerable en el tema accidentabilidad, el objetivo de la investigación es analizar el conocimiento que tiene esta población en estudio sobre lo que es Educación Vial. Se aplicó un enfoque cuantitativo, los tipos de investigación utilizadas fueron bibliográfica y descriptiva, apoyada en los métodos inductivo-deductivo y analítico-sintético y deductivo; las técnicas utilizadas para recopilar información primaria corresponden a la encuesta aplicada a los estudiantes de bachillerato. Los resultado de la investigación evidenciaron que dicha población es un ente que tiene bajos conocimientos de lo que es Educación Vial, es así que no existe una capacitación adecuada en los centros educativos sobre este tema tan vital, así como un desinterés por mejorar esta algia situación, debilidades con una falta de señalética adecuada. Para superar dichas falencias, es imperiosa la necesidad de implementar una asignatura sobre educación vial en los diferentes niveles de educación diseñar estrategias comunicacionales, con el propósito de concientizar a toda la ciudadanía sobre el tema de Primero el Peatón, con la finalidad de generar identidad y proyectar una imagen favorable y así disminuir las elevadas tasas de accidentabilidad que se tiene en el país.

Palabras claves: Educación vial, Análisis Estadístico, Accidentes de tránsito, Señalética

\section{Introducción.}

Los accidentes de tránsito es una de las problemáticas más comunes en la actualidad ya que los vehículos son uno de los medios de transporte más utilizados en estos tiempos, los cuales son indispensables para trasladarse de un lugar a otro. Veremos que muchas veces la imprudencia de las personas, el consumo del alcohol, desconocimiento sobre la educación vial, falta de control por parte de las autoridades hace que se incremente día a día los índices de accidentes de tránsito.

A nivel nacional la tasa de fallecido respecto al número de siniestros ha aumentado en el primer semestre del 2018, respecto al mismo período de los años 2014, 2015, 2016 y 2017, según estadísticas de la Agencia Nacional de Tránsito (ANT). 
Por ello la educación vial se concibe como parte fundamental de la formación y educación ciudadana, en la promoción de una cultura de valoración y respeto por la propia vida y la de los demás. La educación vial cobra mayor relevancia cuando se observa la alta tasa de accidentes de tránsito que se producen diariamente.

La falta de educación vial es la principal causa de los accidentes de tráfico se sitúa por delante del mal estado de las carreteras o el carné por puntos. Las fallas humanas están presentes en el $95 \%$ de accidentes de tránsito y la principal causa de los siniestros, en un $58 \%$, es la impericia del conductor, es decir, su falta de experiencia o habilidad. En el 2013, según datos de la ANT disponibles hasta septiembre en su sitio web, la impericia del conductor fue la causante del $51 \%$ de accidentes y hasta noviembre del 2014 sigue siendo la principal, con el $35 \%$.

Los accidentes, según el informe de los observatorios, han dejado entre el 2008 y 2013 un promedio anual de 51.000 heridos y 4.800 muertos, es decir, 13 por día o uno cada dos horas.

Según datos de la ANT, a noviembre del 2014, el $85 \%$ de vehículos involucrados en siniestro corresponde a automóviles (41\%), motos (19\%), camionetas (14\%) y jeeps (11\%), cuya operación requiere de licencia no profesional. El resto son buses $(6 \%)$, camiones (4\%) y otros pesados $(5 \%)$ que, sin embargo, generan la mayor cantidad de muertos. (Universo, 2014)

Po lo cual Riobamba incluyo la campaña denominada "La vida no es un accidente" con el objetivo de disminuir los accidentes de tránsito en el cantón, que, hasta octubre de 2016, ubicaba a la cabeza de la lista de ciudades con un índice del 19\%. El más alto de mortalidad vial a nivel nacional.

"La campaña pretende crear una cultura vial, tanto en las señaléticas como en viabilidad y normas básicas de conducción”, manifestó una funcionaria de la ANT Riobamba. También resaltó que las capacitaciones son clave fundamental en este proceso, ya que han permitido que la ciudadanía se informe, conozca y transmita sus conocimientos. Estudios técnicos revelaron que la principal causa de accidentes se debía a la ausencia de señalética de tránsito en las intersecciones de la urbe. (Telegrafo, 2017)

\section{Metodología}

Según los objetivos formulados se empleó un enfoque cuantitativo, una investigación de tipo Descriptiva, se aplicaron los métodos inductivo-deductivo, analítico-sintético. Al comenzar dicha investigación iniciamos por recolectar datos en las Unidades Educativas a estudiantes de bachillerato de la ciudad de Riobamba, la cual se nos fue facilitada del Ministerio de Educación, después se procedió a realizar la zonificación por parroquias de la ciudad. 
Y así poder determinar deliberadamente que centros educativos (bachillerato) se encuentran cerca de las mayores zonas de tráfico vehicular y su número de población para escoger los establecimientos que serán parte de la muestra a estudio y contabilizar cuantas encuestas se realizara a cada institución dependiendo de su población.

Cabe recalcar que para que la información sea más real y precisa de escogió por lo menos a una institución por parroquia, de igual forma se extrajo una muestra de unidades educativas, fiscales y fiscomisiónales

\section{Resultados}

Tabla 1.- Conoce acerca de educación vial

\begin{tabular}{ccc}
\hline SI & NO & TOTAL \\
\hline $42 \%$ & $58 \%$ & $100 \%$ \\
\hline
\end{tabular}

Fuente: Encuesta.

Fuente: Trabajo de campo

Con respecto al concociemitno a educación Vial que los estudiantes tienen mas del 50\% descoconocen la temática como tal, lo que nos lleva a colaborar que las instituciones educativas no imparten ningún conocimiento sobre el tema y si cierto porcentaje conoce es por los campos de acción que se imparte en el segundo año de bachillerato.

\section{Tabla 2: El adecuado desenvolvimiento en las vías depende de la educación vial}

\begin{tabular}{ccc}
\hline SI & NO & TOTAL \\
\hline $86 \%$ & $14 \%$ & $100 \%$ \\
\hline
\end{tabular}

Fuente: Encuesta.

Elaboración: Entidad ejecutora

Casi en su totalidad lo estudiantes reconocen que el desenvolvimiento en las vías va de la mano con los conocimientos que se tenga o se adquiera de educación vial un mínimo porcentaje cree que no y esto lo justifican a que depende solo de la distracción que tenga el peatón en las mismas. 
Tabla 3: Dentro de la ciudad existe una adecuada señalética

\begin{tabular}{ccc}
\hline SI & NO & TOTAL \\
\hline $40 \%$ & $60 \%$ & $100 \%$ \\
\hline
\end{tabular}

Fuente: Encuesta.

Elaboración: Entidad ejecutora

Respecto a la existencia de señalética en ciudad un $60 \%$ de estudiantes responde que esta no es la más adecuada, por lo que se debería se debería exigir a las autoridades competentes colocar señalización en las vías de la ciudad.

Tabla 4: Se debe capacitar a peatones y conductores acerca de educación vial

\begin{tabular}{ccc}
\hline SI & NO & TOTAL \\
\hline $90 \%$ & $10 \%$ & $100 \%$ \\
\hline
\end{tabular}

Fuente: Encuesta.

Elaboración: Entidad ejecutora

El 90\% de los entrevistados creen que se debe dar una capacitación a conductores y peatones sobre Educación Vial además manifiestan que esto debe ir acompañado de charlas de concientización y así poder evitar y prevenir accidentes de transito.

Tabla5: Medio de transporte que utiliza

\begin{tabular}{cc}
\hline TAXI & $10 \%$ \\
\hline CARRO PARTICULAR & $21 \%$ \\
BUSETA & $6 \%$ \\
A PIE & $17 \%$ \\
BUS & $42 \%$ \\
BICICLETA & $4 \%$ \\
TOTAL & $100 \%$
\end{tabular}

Fuente: Encuesta.

Elaboración: Entidad ejecutora 
En movilidad es importante conocer el medio más utilizado para movilizarse, es así que un $75 \%$ de los peatones y/o usuarios se movilizan en transporte motorizado lo cual es significativo para que exista una mayor taza de accidentabilidad.

Tabla 6: Usted ha sufrido algún tipo de accidente fuera del establecimiento educativo.

\begin{tabular}{ccc}
\hline SI & NO & TOTAL \\
\hline $73 \%$ & $27 \%$ & $100 \%$
\end{tabular}

Fuente: Encuesta.

Elaboración: Entidad ejecutora

Mas del 70\% de los estudiantes han sufrido accidentes fuera de las unidades educativas otra razón por cual debemos focalizarnos en impartir conocimientos sobre Educación Vial y disminuir paulatinamente los accidentes fuera de las instituciones educativas, de modo que padres y educandos se sientan seguros.

Tabla 7: Las autoridades promueven conocimientos de educación vial

\begin{tabular}{ccc}
\hline $\mathrm{SI}$ & NO & TOTAL \\
\hline $62 \%$ & $38 \%$ & $100 \%$ \\
\hline
\end{tabular}

Fuente: Encuesta.

Elaboración: Entidad ejecutora

Respecto a las autoridades de las diferentes unidades educativas si estas promueven el conocimiento sobre Educación Vial, los entrevistados en un 62\% que no, mientras que $38 \%$ manifiesta que no donde además aseveran que en cierta forma son solo charlas y no capacitaciones como se debería hacer para que un cien por ciento los estudiantes manifiesten e prenden el tema de Educación Vial

\section{Discusión}

Según lo investigado los estudiantes de bachillerato de la ciudad de Riobamba se pronuncian en forma mayoritaria sobre una capacitación que se debe exponer a peatones y conductores en temas relacionados a educación vial, lo cual se coincide con las aseveraciones que manifiesta Madero en su investigación "Sistema de Transito y Seguridad Vial del Ecuador Modelo de Gestión". Ante lo cual estoy seguro que la capacitación a peatones y conductores seria unos de los puntos más importantes a adoptarse con el propósito de tener un adecuado conocimiento en lo que es Educación Vial. 
Por otra parte la investigación también toma como otro punto a ser considerado, el de adoptar una materia sobre educación vial en la malla curricular a nivel de bachillerato. Según otras investigaciones consideran que la asignatura debe ser considerada en todo nivel de educación, es así como lo manifiesta (Foot \& Chapman et al., 1990). Sobre estas aseveraciones podemos considerar que el hecho de considerarse como una asignatura a la Educación Vial dentro de la malla curricular es algo urgente y necesario para disminuir la tasa de accidentabilidad en el país.

El hecho de utilizar automóviles, trasporte público y/o privado para movilizarnos en un gran porcentaje ha hecho que la taza de accidentabilidad también se incremente, Por otra parte las Naciones Unidas declararon que entre los años 2011 y 2020 el decenio de Acción para la Seguridad Vial, según estos hechos consideramos importante de realizar campañas más periódicas de concientización sobre el "Respeto al Peatón y a las Señales de Tránsito", con el único fin de no seguir incrementando los accidentes de tránsito.

\section{Conclusiones.}

- Tras un profundo análisis del objetivo general y específicos previamente enunciados, en los antecedentes e introducción procedemos a resaltar que cada uno de ellos se desarrolló de forma adecuada, teniendo como resultado un $100 \%$ del cumplimiento de los mismos.

- A partir del análisis de problemáticas y la contextualización de la teoría relacionada al presente trabajo de investigación, podemos concluir que la falta de conocimiento sobre educación vial a temprana edad es un factor que interviene en para que exista un alto nivel de accidentes de tránsito.

- Del análisis realizado en la presente investigación, así como de la información y datos levantados, se desprenden que se debe implementar una materia sobre educación vial en las unidades educativas basándose en los resultados obtenidos en campo de que $86 \%$ aceptarían recibir esta materia y $14 \%$ se opondrían, para así poder cambiar la forma de actuar y pensar de la juventud, a través del apoyo de las autoridades que están de acuerdo con la noción.

\section{Referencias bibliográficas.}

Anonimo. (2014). CONCEPTO.DEFINICION.DE. Obtenido de Definicion de educacion Vial: https://conceptodefinicion.de/educacion-vial/

Anonimo. (s.f.). e-mediadrive. Obtenido de ¿Qué es seguridad vial?: http://culturavial.com/seguridad-vial/que-es-seguridad-vial.html

CTE. (2014). Comisión de Tránsito del Ecuador. Obtenido de Conozca - Señales de Tránsito: http://www.comisiontransito.gob.ec/conozca-senales-de-transito/ 
Orozco, G. M. (s.f.). Prueba de ruta.com. Obtenido de Tipos de accidentes de tránsito: https://www.pruebaderuta.com/tipos-de-accidentes-de-transito.php

Porras, L. L. (Agosto de 2012). UNIVERSIDAD DE GUAYAQUIL. Obtenido de Analisis de la Educacion vial en las escuelas fiscales de la Orquideas con el proposito de creacion de talleres didacticos los para estudiantes: http://repositorio.ug.edu.ec/bitstream/redug/2467/1/TRABAJO\%20DE\%20GRADO.pdf

Telegrafo, E. (19 de marzo de 2017). Campaña vial en Riobamba redujo de 100 a 89 accidentes de tránsito. El Telegrafo, pág. 24.

Universo, E. (14 de 12 de 2014). Mayoría de accidentes en Ecuador ocurre por falta de experiencia del conductor. El Universo, pág. 33. 


\section{PARA CITAR EL ARTÍCULO INDEXADO.}

Londo Yachambáy, F., Sánchez Salcán, N. de J., Morales Noriega, M., \& Guerra Sarche, X. (2019). Estudio estadístico de la educación vial en las Unidades Educativas de la Ciudad de Riobamba. Ciencia Digital, 3(3), 195-203. https://doi.org/10.33262/cienciadigital.v3i3.624

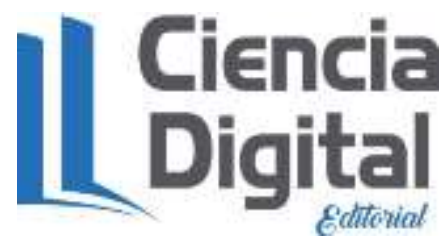

El artículo que se publica es de exclusiva responsabilidad de los autores y no necesariamente reflejan el pensamiento de la Revista Ciencia Digital.

El artículo queda en propiedad de la revista y, por tanto, su publicación parcial y/o total en otro medio tiene que ser autorizado por el director de la Revista Ciencia Digital.
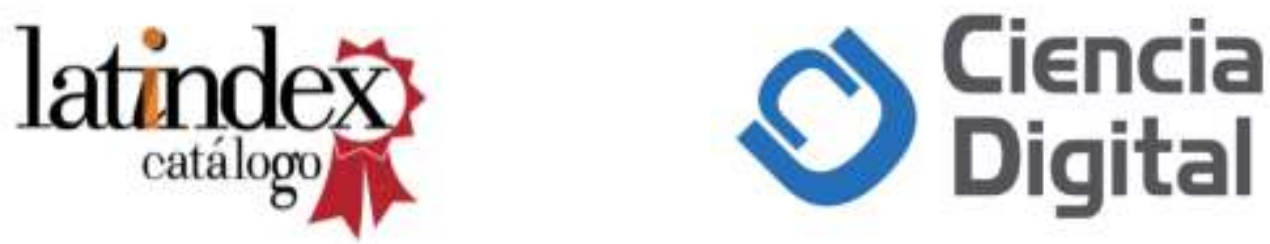